\title{
CALIDAD MOLINERA DE LAS VARIEDADES DE ARROZ SD20A Y PAYARA 1FL, Y SU RESISTENCIA A Sitophilus oryzae (L.) Y Rhyzopertha dominica (F.)
}

\section{MILLING QUALITY OF RICE VARIETIES SD20A AND PAYARA 1FL, AND THEIR RESISTANCE TO Sitophilus oryzae (L.) AND Rhyzopertha dominica (F.)}

\author{
Laury Rangel ${ }^{1}$, Yohan Solano Rojas", Dilcia Hernández ${ }^{3}$, Marlene De Padua $^{4}$, José Morales Sánchez ${ }^{5}$
}

\begin{abstract}
${ }^{1}$ Ingeniero Agroindustrial. Universidad Centroccidental Lisandro Alvarado, Portuguesa, Venezuela, e-mail: laury_710@hotmail.com, (iD https://orcid.org/0000-0002-2701-5515; ${ }^{2}$ Ingeniero Agrónomo, M.Sc. en Entomología. Universidad Centroccidental Lisandro Alvarado, Lara, Venezuela, e-mail: ysolano@ucla.edu.ve, (iDhttps://orcid.org/0000-0001-9762-2815; ${ }^{3}$ Doctora en Entomología. Universidad Centroccidental Lisandro Alvarado, Lara, Venezuela, e-mail: dilciahernandez@ucla.edu.ve, (iD https://orcid.org/0000-0001-5813-4982; ${ }^{4}$ Ingeniero Agroindustrial, M.Sc. Ciencia de los Alimentos. Universidad Centroccidental Lisandro Alvarado, Lara, Venezuela, e-mail: mrpadua@gmail.com, (iDhttps://orcid.org/0000-0002-5555-4479; ${ }^{5}$ Ingeniero Agrónomo, M.Sc. en Entomología. Universidad Centroccidental Lisandro Alvarado, Lara, Venezuela, e-mail: jmorales@ ucla.edu.ve, (Dhttps://orcid.org/0000-0002-2125-5905. *autor de correspondencia: ysolano@ucla-edu-ve
\end{abstract}

Rev. U.D.C.A Act. \& Div. Cient.21(2):351-357, Julio-Diciembre, 2018 https://doi.org/10.31910/rudca.v21.n2.2018.980

Artículo de acceso abierto publicado por Revista U.D.C.A Actualidad \& Divulgación Científica bajo una licencia Creative Commons CC BY-NC 4.0

\section{RESUMEN}

La agroindustria requiere de variedades de arroz, que permitan obtener granos enteros durante su procesamiento y que resistan el ataque de insectos. En este estudio, se determinó la calidad molinera de las variedades SD20A y Payara 1FL y su resistencia a Sitophilus oryzae (L.) y Rhyzopertha dominica (F.). En la primera fase, se calculó el índice de blancura (IB) y rendimiento en molino (RM) de ambas variedades, mientras que, en la segunda, fueron sometidas a los ataques de $S$. oryzae y $R$. dominica, en su condición de arroz paddy y pulido, durante tres meses. La resistencia a los insectos, se interpretó con base al número de adultos emergidos al finalizar el periodo de evaluación. Hubo diferencias estadísticas $(p<0,01)$ entre el IB y el RM de las variedades de arroz SD20A $\left(32,40 \pm 1,87^{\circ}\right.$ Kett y $\left.44,40 \pm 2,55 \%\right)$ y Payara $1 F L$ $\left(35,90 \pm 0,63^{\circ}\right.$ Kett y $\left.48,20 \pm 1,69 \%\right)$, respectivamente. El número de adultos de $S$. oryzae y $R$. dominica fue diferente entre SD20A y Payara 1FL $(p<0,01)$, siendo mayores los valores registrados para las variedades en condición de arroz pulido que sobre arroz paddy. La cantidad de adultos de $R$. dominica fue siempre superior a la de $S$. oryzae, excepto para el arroz pulido de Payara 1FL, donde no hubo diferen- cias significativas $(p>0,01)$. Los resultados muestran que Payara 1FL presentó mayor RM que SD20A y mostró mayor resistencia a los ataques de $S$. oryzae que a los de $R$. dominica.

Palabras clave: arroz, plagas, resistencia a los insectos.

\section{ABSTRACT}

Agroindustry requires rice varieties that allow obtaining whole grains during its processing and also to resist insect attacks. In this study was determined milling quality of varieties SD20A and Payara 1FL, and their resistance to Sitophilus oryzae (L.) and Rhyzopertha dominica (F.). In the first phase, the whiteness ratio (WR) and grain yield in a mill (GY) were calculated for both varieties, while in the second phase, those were through the attack of $S$. oryzae and $R$. dominica, in paddy and milled rice condition, during three months. The resistance was interpreted based on the number of adults emerged at the end of the experiment. There were statistical differences $(p<0.01)$ between WR and GY of rice varieties SD20A (32.40 $\pm 1.87^{\circ}$ Kett and $\left.44.40 \pm 2.55 \%\right)$ and Payara $1 \mathrm{FL}\left(35.90 \pm 0.63^{\circ} \mathrm{Kett}\right.$ and $\left.48.20 \pm 1.69 \%\right)$. Adult emer- 
ged numbers of $S$. oryzae and $R$. dominica was different between SD20A and Payara 1FL $(p<0.01)$, being higher in milled rice than those registered on paddy rice. The amount of adult of $R$. dominica was always higher than $S$. oryzae, except for Payara 1FL milled rice in where no significative difference $(p>0.01)$. Results showed that Payara 1FL had a higher yield in the mill than SD20A, and higher resistance to $S$. oryzae attacks than to $R$. dominica.

Keywords: rice, pest, insect resistance.

\section{INTRODUCCIÓN}

Las variedades de arroz destinadas al uso agroindustrial deben ser de alta calidad molinera, es decir, que garanticen la obtención del mayor porcentaje de granos enteros (Acevedo et al. 2004; Castillo, 2009; Pieters et al. 2011). Durante el procesamiento del arroz, el descascarado y el pulimento son las etapas más importantes en la valoración de su calidad, ya que es donde los granos son sometidos a fuerzas de gran magnitud, que provocan la ruptura de aquellos estructuralmente más débiles (León \& Carreres, 2001; Castillo, 2009).

De igual forma, durante el proceso de pulido, se elimina la capa de aleurona de los granos de arroz, la cual, contiene proteínas, lípidos, vitaminas y minerales, que pueden incrementar la susceptibilidad al ataque de insectos, toda vez que ofrece nutrientes para el desarrollo de estas plagas (Juliano, 1981). Es por ello, que las variedades de mayor calidad serán aquellas de granos fuertes, con índice de blancura entre 38 - $40^{\circ}$ Kett (bajo contenido de aleurona) y rendimiento en molino superior a 47\% (COVENIN, 1990; CONARROZ, 2006).

La resistencia de los granos de arroz al ataque de insectos es otra característica deseable en la selección de variedades para uso agroindustrial. En este sentido, varios autores indicaron que variedades resistentes han permitido reducir las pérdidas de arroz paddy o pulido, asociadas a la actividad alimenticia de los insectos, tales como disminución del peso y reducción del valor nutritivo del grano (carbohidratos y proteínas) y la proliferación de hongos (Saini \& Rodríguez, 2004; Alonso et al. 2009; Arthur et al. 2012; Astuti et al. 2013). Las pérdidas ocasionadas por los insectos, se pueden ubicar entre 5 y $10 \%$ en almacenes de arroz (Maqsood et al. 1988; Shafique \& Chaudry, 2007).

El arroz almacenado puede ser afectado por especies, como Sitophilus oryzae L., S. zeamais L. (Coleoptera: Curculionidae), Rhyzopertha dominica F. (Coleoptera: Bostrichidae), Oryzaephilus surinamensis L. (Coleoptera: Cucujidae) Sitotroga cerealella (Olivier) (Lepidoptera: Gelechiidae), cuyas poblaciones suelen ser controladas, utilizando estrategias convencionales, como la aplicación de insecticidas, reducción del contenido de humedad del grano, aireación y control de la temperatura, manejo de las condiciones de la estructura de almacenamiento (Rashid et al. 2009; Khan \& Halder, 2012; Astuti et al. 2013; Badii et al. 2013) y, no convencionales, como la resistencia de los granos al ataque de insectos. De acuerdo con Smith (2005), esta resistencia puede ser provocada por antibiosis (afectación de la biología, incremento de la mortalidad o reducción de la reproducción y longevidad) o antixenosis (cambios en el comportamiento alimenticio).

Diversas investigaciones han indicado que la resistencia de variedades de arroz al ataque de insectos se ha asociado con alta tasa de mortalidad, baja emergencia de adultos, baja tasa de oviposición, bajo consumo o comportamiento disuasorio de la alimentación de las plagas (Shafique \& Chaudry, 2007; Arthur et al. 2013; Astuti et al. 2013; Costa et al. 2016). Otros estudios han señalado que factores, como fisuras de las brácteas, el grosor de la cáscara, la dureza del grano y el contenido de sílice, fenoles, proteína y amilasa en los granos de arroz, pueden inducir resistencia (Chanbang et al. 2008; Arthur et al. 2012; Ribeiro et al. 2012; Badii et al. 2013; Santos et al. 2015).

En Venezuela, las variedades de arroz SD20A y Payara 1FL son unas de las más utilizadas por los agricultores, debido a que pueden alcanzar rendimientos cercanos a 55 y $64 \%$, respectivamente y son resistentes a plagas, como Tagosodes orizicolus (Muir) (Hemiptera: Delphacidae) y enfermedades ocasionadas por Pyricularia grisea (Ascomycota: Magnaporthaceae) y Rhizoctonia solani (Basidiomycota: Agonomicetaceae), en condiciones de campo (APROSCELLO, 2017). De acuerdo con Acevedo et al. (2004), las variedades de arroz producidas en el país poseen características adecuadas para el procesamiento agroindustrial; sin embargo, el uso de variedades mezcladas en una misma molienda puede afectar su rendimiento, toda vez que las características de los granos de cada variedad ofrecen diferentes niveles de resistencia al descascarado y pulimento (Zeledón et al. 2007; Castillo, 2009), de modo que es importante evaluar la calidad molinera de las variedades, de forma independiente. Esta investigación, se condujo con el objetivo de determinar la calidad molinera de las variedades SD20A y Payara 1FL y su resistencia con relación a la reproducción de $S$. oryzae y $R$. dominica, lo cual, constituye información valiosa para los agricultores y las empresas procesadoras de arroz.

\section{MATERIALES Y MÉTODOS}

Determinación de la calidad molinera de las variedades de arroz SD20A y Payara 1FL: Las muestras de granos de arroz de las variedades SD20A y Payara 1FL fueron suministradas en condición de arroz paddy, por una empresa procesadora del estado Portuguesa (Venezuela) y llevadas al Laboratorio de Biología y Fisiología Poscosecha del Decana- 
to de Agronomía de la Universidad Centroccidental Lisandro Alvarado (UCLA), en el estado Lara (Venezuela), para la determinación de su calidad molinera, siguiendo los parámetros establecidos en la norma COVENIN 44-90 (1990).

Porcentaje de granos enteros y granos partidos: Se seleccionaron aleatoriamente muestras de 100 gramos de arroz para cada variedad. Las muestras fueron homogeneizadas y sometidas a molienda, en un molino de laboratorio (tres en uno), marca Zaccaria, modelo PAZ-1 DTA, en el cual, los granos fueron descascarados, para obtener arroz integral. Seguidamente, el arroz descascarado ingresó a la cámara pulidora del molino, donde perdió el germen y el pericarpio, para obtener arroz blanco. Una vez obtenido, se determinó el porcentaje de granos enteros y granos partidos, de la siguiente manera:

Granos enteros: granos de arroz pulido, cuya longitud es igual o mayor a tres cuartas partes de la longitud del grano normal. Se calculó, a través de la siguiente fórmula:

Porcentaje de granos enteros $=$ (Masa de granos enteros (gramos))/(Masa de muestra limpia (gramos)) x100

Granos partidos: pedazos de granos pulidos, cuyo tamaño sea menor a las tres cuartas partes de la longitud media del grano entero. Se calculó, con la siguiente fórmula:

Porcentaje de granos partidos $=$ (Masa de granos partidos $($ gramos))/(Masa de muestra limpia (gramos)) x100

Índice de blancura (IB): Los granos enteros obtenidos para cada variedad fueron utilizados para determinar el índice de blancura ('Kett). Las muestras analizadas consistieron en $16 \mathrm{~g}$ de granos enteros, introducidos en un medidor de blancura marca Zaccaria, modelo MBZ-1. Se usó, como parámetro de IB, los valores comprendidos entre $38-40^{\circ} \mathrm{Kett}$, de acuerdo con lo establecido por CONARROZ (2006).

Rendimiento en molino: El rendimiento, se expresó como la relación porcentual en masa de granos enteros de arroz blanco con respecto al arroz cascara. Se calculó, a través de la siguiente fórmula:

Porcentaje de rendimiento $=($ Porcentaje de granos enteros $\mathrm{x}$ Blanco total $) /(2 \times 100)$

Determinación de la resistencia de las variedades SD20A y Payara 1FL, a S. oryzae y R. dominica

Cría de insectos: El gorgojo del arroz $S$. oryzae y el barrenador menor de los granos $R$. dominica fueron colectados en una empresa procesadora de arroz y llevados al Laboratorio de Biología y Fisiología Poscosecha de la UCLA. En el laboratorio, cuatro grupos de 50 adultos de cada especie fueron transferidos a ocho envases de vidrio de 3,8L de capacidad, respectivamente. En cada envase, se colocaron $500 \mathrm{~g}$ de arroz, como fuente de alimento para los insectos y se cubrió con tela organdí, para permitir la ventilación y evitar el escape de los gorgojos.

Los envases fueron llevados a una sala de cría, a $25 \pm 2^{\circ} \mathrm{C}$ y $72 \pm 10 \%$ HR y un fotoperiodo de 12:12 (L:D). Los adultos parentales fueron removidos a los 15 días y, diariamente, cada envase fue observado, para conocer el estado de desarrollo de la cría, hasta obtener la primera generación (F1). Una vez obtenidos los adultos de la F1, se conformaron 80 grupos (40 para cada especie de insecto), de 30 individuos, no sexados y con edades comprendidas entre 14 y 21 días, que se usaron en el experimento de resistencia.

Experimentos de resistencia a insectos plaga: Las variedades de arroz en condición de paddy fueron sometidas a esterilización bajo refrigeración, a temperatura de $-5^{\circ} \mathrm{C}$, durante 1 día. A continuación, los granos fueron esparcidos sobre un papel absorbente en una bandeja plástica, por un periodo de 24 horas, a $25^{\circ} \mathrm{C}$ y $65 \% \mathrm{HR}$, con la finalidad de disminuir la humedad adquirida en el refrigerador. Seguidamente, los mismos fueron colocados en una estufa para reducir su humedad, a valores comprendidos entre $12-14 \%$, lo cual, fue comprobado con ayuda de una balanza Steinlite SB 900. El arroz pulido de ambas variedades fue obtenido directamente de la planta procesadora, con humedad de $12 \%$.

El experimento, para determinar la resistencia de las variedades de arroz SD20A y Payara 1FL en contra de S. oryzae y $R$. dominica, fue realizado utilizando granos de arroz entero de las variedades en ambas condiciones de procesamiento (paddy y pulido). Previamente, se removieron granos partidos, agrietados, sin gluma, lema o palea y la materia extraña. Seguidamente, se pesaron muestras de $40 \mathrm{~g}$ de arroz con ayuda de una balanza electrónica, Marca KERN, Modelo EMB220-1, colocadas en envases plásticos de $60 \mathrm{~mL}$ de capacidad, con la tapa perforada y cubierta con tela organdí, para permitir la ventilación.

A cada muestra de arroz, se introdujeron 30 adultos de $S$. oryzae o $R$. dominica, de acuerdo con cada experimento y se mantuvieron en salas de cría, con las condiciones descritas anteriormente. En la tercera semana, posterior al inicio del experimento, se extrajeron los adultos introducidos inicialmente; el primer conteo de adultos emergidos comenzó a partir de la cuarta semana y semanalmente, durante tres meses, de manera simultánea, en cada variedad y condición de procesamiento. Después de cada conteo, los adultos fueron descartados de los envases. La resistencia de las variedades de arroz, se consideró como un efecto de antibiosis, relacionado con la reducción del número de insectos que emergieron, durante el periodo de evaluación. 
Análisis estadístico: Los datos obtenidos para la determinación de la calidad molinera fueron analizados por medio de estadística descriptiva con 10 repeticiones. El experimento de resistencia tuvo un diseño completamente al azar, con 10 repeticiones por tratamiento y sus datos se procesaron en el programa Statistix 10.0. Se utilizaron pruebas de comparación de medias LSD, con un nivel de confianza de $99 \%$.

\section{RESULTADOS Y DISCUSIÓN}

Calidad molinera de las variedades de arroz SD20A y Payara 1FL: Las variedades de arroz SD20A y Payara 1FL mostraron un comportamiento estadísticamente diferente en calidad molinera, donde Payara 1FL se ajustó mejor a las normas COVENIN 44-90 (1990) (Tabla 1). Con relación al porcentaje de granos enteros, ambas variedades registraron valores similares, mientras que el porcentaje de granos partidos fue mayor en SD20A que en Payara 1FL.

Con relación al IB, fue mayor en Payara 1FL que en SD20A (Tabla 1); no obstante, los valores obtenidos se encuentran por debajo del rango estipulado por CONARROZ (2006). Al respecto, Zeledón \& Corrales (1997) indicaron que la etapa de pulido es la que permite obtener un mayor o menor IB en los granos de arroz, ya que durante el proceso se remueve la capa de aleurona y se eliminan pequeñas partículas adheridas al grano, por lo que, a menor IB mayor la cantidad de aleurona remanente en el grano.

El rendimiento en molino fue estadísticamente diferente entre las variedades $(p<0,01)$ (Tabla 1), siendo Payara 1FL la que mostró mejor comportamiento, de acuerdo con lo contemplado por la norma COVENIN 44-90 (1990), donde se estipula que el rendimiento debe ser superior de $47 \%$. Diversos investigadores han indicado que el rendimiento en molino puede estar influenciado por el manejo agronómico (fertilización, manejo de riego, maduración de grano), el clima y factores industriales (secado, almacenamiento y molienda) (León \& Carreres, 2001; Castillo, 2007). Zeledón et al. (2007) señalaron que los granos más delgados suelen ser los que se quiebran en mayor proporción, en consecuencia, muestran menor rendimiento en molino, lo cual, pudo haber ocurrido con la variedad SD20A.

Con base en los resultados obtenidos, se infiere que la variedad SD20A mezclada con Payara 1FL, puede inducir una reducción en el rendimiento en molino cuando se les procese de forma combinada, debido a que las características de los granos de cada variedad pueden ofrecer diferentes niveles de resistencia al descascarado y pulimento (Castillo, 2009). Al respecto, Zeledón et al. (2007) indicaron que la mezcla de variedades de arroz CR 113 y CR 5272 produjo un efecto perjudicial en el rendimiento en molino, que no se registró cuando las variedades se procesaron independientemente.

Resistencia de las variedades de arroz SD20A y Payara $1 \mathrm{FL}$, a $\mathrm{S}$. oryzae y $\mathrm{R}$. dominica: El número de adultos emergidos de las especies $S$. oryzae y $R$. dominica fue estadísticamente diferente entre las variedades SD20A y Payara $1 \mathrm{FL}(p<0,01)$, siendo mayores los valores obtenidos para la condición de arroz pulido que los registrados sobre arroz paddy (Tabla 2). La cantidad de adultos emergidos de $R$. dominica fue siempre superior a la de $S$. oryzae, excepto para la condición de arroz pulido de la variedad Payara 1FL, donde no hubo diferencias significativas entre las especies de insectos $(p>0,01)$.

Los resultados obtenidos en esta investigación muestran que las variedades de arroz SD20A y Payara 1FL ofrecieron mayor resistencia al ataque del gorgojo del arroz, que al del barrenador menor de los granos, tanto en la condición de arroz paddy como en la de arroz pulido. De acuerdo con Shafique \& Chaudry (2007), las diferencias en el número de insectos que se desarrollan en granos de distintas variedades reflejan un efecto de antibiosis del alimento. Otras investigaciones, también señalan que, diferencias en la pérdida de peso de los granos, la población de los insectos y sus tasas de mortalidad, se han interpretado como niveles de resistencia de

Tabla 1. Calidad molinera de las variedades de arroz SD20A y Payara 1FL.

\begin{tabular}{|l|c|c|}
\hline Tratamiento & SD20A & Payara 1FL \\
\hline Granos enteros (\%) & $61,90 \pm 3,36 \mathrm{a}$ & $64,70 \pm 2,25 \mathrm{a}$ \\
\hline Granos partidos (\%) & $11,60 \pm 2,56 \mathrm{a}$ & $5,50 \pm 1,50 \mathrm{~b}$ \\
\hline Índice de blancura ( ${ }^{\circ}$ Kett) & $32,40 \pm 1,87 \mathrm{~b}$ & $35,90 \pm 0,63 \mathrm{a}$ \\
\hline Rendimiento (\%) & $44,40 \pm 2,55 \mathrm{~b}$ & $48,20 \pm 1,69 \mathrm{a}$ \\
\hline
\end{tabular}

Basado en 10 observaciones. Promedios dentro de una fila seguidos por la misma letra no son significativamente diferentes ( $>$ > 0,01, Prueba LSD). 
Tabla 2. Número promedio de adultos de Sitophilus oryzae y Rhyzopertha dominica emergidos en granos de arroz, de las variedades SD20A y Payara 1FL.

\begin{tabular}{|l|c|c|}
\hline Tratamiento & S. oryzae & $\boldsymbol{R}$. dominica \\
\hline SD20A Paddy & $2,13 \pm 0,99 \mathrm{cB}$ & $29,38 \pm 12,72 \mathrm{cA}$ \\
\hline Payara 1FL Paddy & $2,50 \pm 1,85 \mathrm{cB}$ & $24,63 \pm 7,93 \mathrm{cA}$ \\
\hline SD20A Pulido & $62,38 \pm 20,69 \mathrm{bB}$ & $260,88 \pm 27,33 \mathrm{aA}$ \\
\hline Payara 1FL Pulido & $161,75 \pm 28,12 \mathrm{aA}$ & $139,13 \pm 10,76 \mathrm{bA}$ \\
\hline
\end{tabular}

Basado en 10 observaciones. Promedios dentro de una columna seguidos por la misma letra minúscula y promedios dentro de una fila seguidos por la misma letra mayúscula no son significativamente diferentes ( $p>0,01$, Prueba LSD).

las variedades de arroz a los ataques de las plagas (Rashid et al. 2009; Arthur et al. 2012; Arthur et al. 2013; Astuti et al. 2013).

Badii et al. (2013) demostraron que las variaciones en la susceptibilidad de ocho variedades de arroz a los ataques de $S$. oryzae estuvieron relacionadas con la longitud de los granos, mientras que Santos et al. (2015) argumentaron que las diferencias en los niveles de resistencia de once variedades de arroz a S. oryzae, se debieron a las variaciones fisicoquímicas de los granos y a factores, como las condiciones climáticas, el régimen hídrico y la fertilidad del suelo, lo cual, puedo influir en el contenido de nutrientes de los granos de arroz. Por otra parte, Avilés et al. (2008) indicaron que $R$. dominica es capaz de dañar fácilmente los granos de arroz, debido a sus poderosas mandíbulas. Asimismo, Avilés et al. (2009) señalaron que el mal ajuste de las glumelas de los granos de arroz paddy puede estimular la oviposición de este insecto.

Con base en estas investigaciones, se puede inferir que la alta cantidad de adultos de $R$. dominica, desarrollados sobre ambas variedades, comparada con la de S. oryzae, se debió a sus habilidades alimenticias y al bajo IB registrado para la variedad SD20A. De acuerdo con Juliano (1981), los granos que contienen restos de aleurona, incrementan la susceptibilidad al ataque de insectos, ya que estos residuos ofrecen nutrientes para su desarrollo. Por su parte, Arthur et al. (2012) y Arthur et al. (2013) señalaron que correlacionar los daños por alimentación de plagas, como $R$. dominica y $S$. cerealella, con el rendimiento en molino de diferentes variedades de arroz, puede ser un método útil para evaluar el impacto de los insectos sobre la calidad del arroz, además de constituir un elemento importante dentro de los programas de manejo de plagas de este cereal en almacenamiento.

De forma general, la variedad Payara 1FL presentó mayor índice de blancura y rendimiento en molino que la variedad SD20A. Las variedades de arroz ofrecieron mayor resistencia al ataque del gorgojo que al del barrenador menor de los gra- nos; no obstante, Payara 1FL, en condición de arroz pulido, tuvo una susceptibilidad similar al ataque de ambos insectos.

Agradecimientos: Al Consejo de Desarrollo Científico, Humanístico y Tecnológico (CDCHT) de la Universidad Centroccidental "Lisandro Alvarado" (UCLA). A la Unidad de Investigación del Departamento de Ecología y Control de Calidad (UNIECCA)- UCLA. A los revisores anónimos por sus valiosos aportes y sugerencias. Conflicto de intereses: El manuscrito fue preparado y revisado con la participación de todos los autores, quienes declaramos que no existe ningún conflicto de intereses que ponga en riesgo la validez de los resultados presentados. Financiación: Este estudio fue financiado por la Universidad Centroccidental Lisandro Alvarado.

\section{BIBLIOGRAFÍA}

1. ACEVEDO, M.; ÁlVAREZ, R.; DELGADO, N.; MORENO, O.; REYES, E.; TORREALBA, G. 2004. Mejoramiento genético en cultivo de arroz en Venezuela. Editorial Instituto Nacional de Investigaciones Agrícolas. Primera Edición. (Venezuela). 202p.

2. ALONSO, M.; AVILA, J.; CALCAGNO, M. 2009. Los cereales en el trópico suramericano: técnicas modernas de conservación. Editorial CDCHT ULA - Fundación Empresas Polar. (Venezuela).446p.

3. ARTHUR, F.H.; ONDIER, G.O.; SIEBENMORGEN, T.J. 2012. Impact of Rhyzopertha dominica (F.) on quality parameters of milled rice. J. Stored Prod. Res. (England). 48:137-142. https://doi.org/10.1016/j. jspr.2011.10.010

4. ARTHUR, F.H.; STARKUS, L.; SMITH, C.M.; PHILLIPS, T.W. 2013. Methodology for determining susceptibility of rough rice to Rhyzopertha dominica and Sitotroga cerealella. J. Pest Sci. (Germany). 86(3):499505. https://doi.org/10.1007/s10340-013-0481-2 
5. ASOCIACIÓN DE PRODUCTORES DE SEMILLA CERTIFICADA DE LOS LLANOS OCCIDENTALES -APROSCELLO-. 2017. Página principal. Disponible desde Internet en: http://www.aproscello.com/?aproscello|-una-organizacion-al-servicio-del-sector-agroalimentario-rif:-j-08507040-0\&id=home (con acceso el 03/06/2017).

6. ASTUTI, L.P.; MUDJIONO, G.; RASMINAH, S.; RAHARDJO, B.T. 2013. Susceptibility of milled rice varieties to the lesser grain borer (Rhyzopertha dominica F.). J. Agric. Sci. (United Kingdom). 5(2):145-149. https:// doi.org/10.5539/jas.v5n2p145

7. AVILÉS, R.; SOTOMAYOR, E.; GUIBERT, G.; MARTINEZ, Y. 2008. Ciclo biológico de Rhyzopertha dominica (F.) en semillas de arroz sometidas a cuatro temperaturas. Fitosanidad (Cuba). 12(4):221-225.

8. AVILÉS, R.; SOTOMAYOR, E.; GUIBERT, G.; MARTÍNEZ, Y. 2009. Vulnerabilidad de la semilla de arroz al ataque de Rhyzopertha dominica (F.) (Coleoptera: Bostrichidae). Fitosanidad. (Cuba). 13(1):7-10.

9. BADII, B.; ASANTE, K.; ADARKWA, C. 2013. Varietal differences in the susceptibility of new rice for Africa (NERICA) to Sitophilus oryzae L. (Coleoptera: Curculionidae). Afr. J. Agric. Res. (Nigeria). 8(16):13751380. https://doi.org/10.5897/AJAR12.2093

10. CASTILLO, A. 2007. Molinería (Pilado) de arroz. Molinería en los trópicos. Ediagro LDTA. Tomo II. Colombia. 308p.

11. CASTILLO, A. 2009. Almacenamiento de granos. Ediagro LDTA. Cuarta Edición. Colombia. 388p.

12. CHANBANG, Y.; ARTHUR, F.H.; WILDE, G.E.; TRONE, J.E. 2008. Hulls characteristics as related to susceptibility of different varieties of rough rice to Rhyzopertha dominica (F.) (Coleoptera: Bostrichidae). J. Stored Prod. Res. (England). 44(3):205-212. https:// doi.org/10.1016/j.jspr.2007.10.003

13. COMISIÓN VENEZOLANA DE NORMAS INDUSTRIALES -COVENIN-. 1990. Norma para arroz paddy 44 - 90. Caracas, Venezuela. Disponible desde Internet en: http://www.sencamer.gob.ve/sencamer/ normas/44-90.pdf (con acceso el 17/04/2017).

14. CORPORACIÓN ARROCERA NACIONAL COSTA RICA CONARROZ-. 2006. Parámetros de calidad molinera. Fundarroz - Venezuela. Disponible desde Internet en:
http://www.conarroz.com/pdf/9Parametrosmolineria. pdf (con acceso el 03/06/2017).

15. COSTA, D.C.; SOUSA, A.C.; SILVA, M.; HEINRICHS, E.A.; LACERDA, M.C.; FREITAS, J.A.; GONÇALVES, F. 2016. Resistance of rice varieties to Sitophilus oryzae (Coleoptera: Curculionidae). Fla. Entomol. (United States). 99(4):769-773. https://doi. org/10.1653/024.099.0432

16. JULIANO, B.O. 1981. Rice grain properties and resistance to storage insects: a review. IRRI (Philippines). 56:1-9.

17. KHAN, H.; HALDER, P. 2012. Susceptibility of six varieties of rice to the infestation of rice weevil Sitophilus oryzae (L.) (Coleoptera: Curculionidae). Dhaka Univ. J. Biol. Sci. (Bangladesh). 21(2):163-168. http://dx. doi.org/10.3329/dujbs.v21i2.11514

18. LEÓN, J.; CARRERES, R. 2001. La calidad de molienda del arroz y el rendimiento de grano entero. Vida Rural (España). 127:38-42.

19. MAQSOOD, I.; KHATTAK, S.U.; KHALIL, S.K.; HUSSAIN, N.; HAMED, M. 1988. Combined infestation and losses caused by three storage insects in Pak-81 wheat variety. The Nucleus (Pakistan). 25:23-26.

20. PIETERS, A.; GRATEROL, E.; REYES, E.; ÁLVAREZ, R.; GONZÁLEZ, A. 2011. Cincuenta años de mejoramiento genético del arroz en Venezuela. INTERCIENCIA (Venezuela). 36(12):943-948.

21. RASHID, M.H.; HAQUE, M.A.; HUDA, M.S.; RAHMAN, M.M.; AHSAN, A.F. 2009. Study on resistance of different rice varieties against rice weevil, Sitophilus oryzae (L.). Int. J. Sustain. Crop Prod. (Bangladesh). 4(1):35-40.

22. RIBEIRO, C.S.N.; MARTINS, G.V.; GUIMARÃES, J.F.R.; SILVA, E.F. 2012. Resistência de genótipos de arroz a pragas de grãos armazenados. Caatinga (Brasil). 25:183-187.

23. SAINI, E.; RODRÍGUEZ, S. 2004. Insectos perjudiciales a los productos almacenados. INTA. Primera Edición. Buenos Aires. 56p.

24. SHAFIQUE, M.; CHAUDRY, M. 2007. Screening of rice genotypes for resistance to storage insects. Pak. Entomol. (Pakistan). 29(1):19-21. 
25. SMITH, C.M. 2005. Plant resistance to arthropods: molecular and conventional approaches. Editorial Springer. Berlin, Germany. 426p.

26. SANTOS, C.; AGBANGLA, C.; CHOUGOUROU, D.; MISSIHOUN, A.A.; AHANHANZO, C. 2015. Influence of agro ecology on rice varietal resistance to Sitophilus oryzae (Coleoptera: Curculionidae) and Sitotroga cerealella (Lepidoptera: Gelechiidae) in Benin. Am. J. Plant Sci. (United States). 6:28322843. http://dx.doi.org/10.4236/ajps.2015.618280

27. ZELEDÓN, M.; CORRALES, V. 1997. Efecto del tiempo de blanqueado del arroz en la sobrevivencia de Rhy- zopertha dominica, en la remoción de las capas externas y en el porcentaje de grano quebrado. Agron. Costarricense (Costa Rica). 21(2):171-178.

28. ZELEDÓN, M.; BARBOZA, R.; CRUZ, M. 2007. Efecto del beneficiado de mezclas de variedades de arroz sobre variables de rendimiento molinero. Agron. Mesoam. (Costa Rica). 18(1):103-113. https://doi. org/10.15517/am.v18i1.5041

Recibido: Mayo 23 de 2018

Aceptado: Octubre 1 de 2018

Cómo citar:

Rangel, L.; Solano Rojas, Y.; Hernández, D.; De Padua, M.; Morales Sánchez, J. 2018. Calidad molinera de las variedades de arroz SD20A y Payara 1FL, y su resistencia a Sitophilus oryzae (L.) y Rhyzopertha dominica (F.). Rev. U.D.C.A Act. \& Div. Cient. 21(2):351-357. https://doi.org/10.31910/rudca.v21.n2.2018.980 